\title{
High-density lipoprotein enhancement of anticoagulant activities of plasma protein $S$ and activated protein $C$
}

\author{
John H. Griffin, ${ }^{1}$ Kazuhisa Kojima, ${ }^{1}$ Carole L. Banka, ${ }^{2}$ Linda K. Curtiss, ${ }^{2}$ \\ and José A. Fernández ${ }^{1}$ \\ ${ }^{1}$ Department of Molecular and Experimental Medicine, and \\ ${ }^{2}$ Department of Immunology, The Scripps Research Institute, La Jolla, California 92037, USA \\ Address correspondence to: J.H. Griffin, The Scripps Research Institute, SBR-5, 10550 N. Torrey Pines Road, \\ La Jolla, California 92037, USA. Phone: (619) 784-8220; Fax: (619) 784-2243; E-mail: jgriffin@scripps.edu \\ Preliminary reports of this study were presented at the American Society of Hematology meeting in Orlando, Florida, USA (December \\ 6-10, 1996. Blood. 88:523a), and the International Society for Thrombosis and Haemostasis Congress in Florence, Italy (June 6-10, \\ 1997. Thromb. Haemost. 77:399a).
}

Received for publication August 24, 1998, and accepted in revised form November 24, 1998.

\begin{abstract}
Low-density lipoprotein (LDL) and high-density lipoprotein (HDL) cholesterol levels are associated, respectively, with either increased risk or apparent protective effects for atherothrombosis. The ability of purified LDL and HDL to downregulate thrombin formation, a contributor to atherothrombotic processes, was assessed. Purified HDL, but not LDL, significantly enhanced inactivation of coagulation factor Va by activated protein C (APC) and protein S, and HDL stimulated protein S-dependent proteolytic inactivation of Va by APC, apparently due to cleavage at Arg306 in Va. In normal plasma, added HDL enhanced APC/protein S anticoagulant activity in modified prothrombin-time clotting assays. When the anticoagulant potency of HDL was compared with phospholipid (PL) vesicles of well-defined composition using this assay, HDL appeared qualitatively different from PL vesicles because HDL showed only good anticoagulant activity, whereas PL vesicles were rather procoagulant. When 20 normal plasmas were tested using this clotting assay, apoA-I levels correlated with anticoagulant response to APC/protein $S(r=0.47, P=0.035)$, but not with activated partial thromboplastin time-based APC resistance ratios. Because $\mathrm{HDL}$ enhances the anticoagulant protein $\mathrm{C}$ pathway in vitro, we speculate that HDL may help downregulate thrombin generation in vivo and that this anticoagulant action is one of HDL's beneficial activities.
\end{abstract}

J. Clin. Invest. 103:219-227 (1999).

\section{Introduction}

The inverse correlation between plasma high-density lipoprotein (HDL) cholesterol (HDL-C) levels and incidence of coronary artery disease (CAD) is well documented (1-4). Molecular mechanisms responsible for the atheroprotective effects of HDL remain controversial, are most likely multifactorial, and may include, inter alia, reverse cholesterol transport to the liver, protection of low-density lipoprotein (LDL) from oxidative modification, and inhibition of cytokine-induced expression of adhesion molecules (5-10).

Thrombin generation via blood coagulation pathways causing platelet activation and platelet-derived growth factor (PDGF) release, as well as fibrin deposition, may contribute to atherogenesis (11). Elevated plasma lipids appear to be procoagulant and may be associated with either clinically significant thrombosis or elevated hemostatic markers of hypercoagulability $(12-15)$. Little is known about the effects of plasma LDL and HDL on plasma anticoagulant mechanisms that regulate blood coagulation. The protein $\mathrm{C}$ pathway provides a major physiologic anticoagulant mechanism by which the proteolytically active form of the vitamin $\mathrm{K}$-dependent zymogen protein $\mathrm{C}$ inactivates factors $\mathrm{Va}(\mathrm{FVa})$ and VIIIa (FVIIIa) in plasma (16-18). Activated protein C (APC) anticoagulant activity is enhanced by plasma protein $\mathrm{S}$, a vitamin $\mathrm{K}$-dependent nonenzymatic cofactor for APC. Protein $\mathrm{C}$ and protein $\mathrm{S}$ are physiological antithrombotic factors, and a severe deficiency of protein $\mathrm{S}$ or protein $\mathrm{C}$ is associated with life-threatening thrombosis, while heterozygous deficiencies are associated with a mild risk of venous thrombosis $(18,19)$. The abnormality known as APC resistance, which is due to a subnormal anticoagulant response of a patient's plasma to APC, causes a mild hypercoagulable state and is a risk factor for venous thrombosis $(18,19)$ and ischemic stroke $(20,21)$. The factor $\mathrm{V}(\mathrm{FV})$ polymorphism of Arg506Gln that most often causes hereditary APC resistance in venous thrombosis patients is not associated with increased risk for CAD or ischemic stroke $(20,22)$. Abnormalities of thrombomodulin, the cell-surface cofactor for generation of APC by thrombin, may be associated with increased risks for myocardial infarction (23).

For potent anticoagulant activity, APC and protein $S$ require negatively charged phospholipids (PL) and $\mathrm{Ca}^{++}$ (24-26). Therefore, this in vitro study was undertaken to examine the influence of purified HDL and LDL on the anticoagulant activities of APC and protein S. Using purified reagents and a novel clotting assay, we show that HDL is an anticoagulant cofactor that potentiates the protein 
C pathway. We speculate that this activity may help explain the beneficial properties of HDL against CAD.

\section{Methods}

Reagents. LDL (density $=1.019-1.063 \mathrm{~g} / \mathrm{ml}$ ) and HDL (density $=1.063-1.21 \mathrm{~g} / \mathrm{ml}$ ) were isolated from normal human plasma (nonsmoking healthy adult males) by sequential density gradient ultracentrifugation in the presence of protease inhibitors and antioxidants and then stored in DMEM, $0.3 \mathrm{mM}$ EDTA at $4^{\circ} \mathrm{C}$ as described $(27,28)$. Lipoprotein concentrations were expressed based on either protein- (29) or choline-containing PL concentrations (Phospholipids B kit; Wako Chemicals, Germany), and apoA-I antigen was measured using an ELISA kit (AlerCHEK Inc., Portland, Maine, USA).

Blood was obtained from routine venipuncture from 20 healthy adult volunteers ( 11 females and 9 males) after overnight fasting, then mixed with $0.129 \mathrm{M}$ sodium citrate (one part plus nine parts blood). Plasma was prepared by centrifugation at $2,000 \times g$ for 20 min at room temperature and then stored at $-80^{\circ} \mathrm{C}$.

Human factor $\mathrm{Xa}(\mathrm{FXa})$ and protein $\mathrm{S}$ were purchased from Enzyme Research Laboratory (South Bend, Indiana, USA). Variant Gln506-FV, normal FV, and prothrombin were purified from plasma $(30,31)$, and variant and normal FV were converted by thrombin to FVa as described $(30,32)$. The thrombin amidolytic substrate, CBS 34-47, was obtained from American Bioproducts (Parsippany, New Jersey, USA); Innovin (recombinant human tissue factor reagent) from Baxter-Dade, (Miami, Florida, USA); bee venom and Naja mossambica mossambica phospholipase $\mathrm{A}_{2}$ from Sigma Chemical Co. (St. Louis, Missouri, USA); and FV-deficient plasma from George King Biomedical (Overland Park, Kansas, USA). Specific antiserum to the heavy chain of FVa and purified human APC were gifts from A. Gale and A. Gruber (The Scripps Research Institute).

Normal LDL and HDL plasma concentrations (defined as 1 $\mathrm{U} / \mathrm{ml}$, or as $100 \%$ ) were considered equivalent to a choline-containing PL content of $0.94 \mathrm{mM}$ and to protein concentrations of $0.75 \mathrm{mg} / \mathrm{ml}$ and $1.5 \mathrm{mg} / \mathrm{ml}$, respectively.

PL vesicles. Purified PLs - phosphatidylethanolamine (PE), phosphatidylserine (PS), phosphatidylcholine (PC) (Avanti Polar Scientific, Alabaster, Alabama, USA) - in chloroform were mixed at various weight ratios and then dried under nitrogen in siliconized glass tubes. After reconstituting the dried material in Tris-buffered saline (TBS) (50 mM Tris, $100 \mathrm{mM} \mathrm{NaCl}, 0.02 \%$ $\mathrm{NaN}_{3}, \mathrm{pH}$ 7.4), the PL-vesicle suspensions, in tubes surrounded by wet ice, were sonicated for six cycles of $30 \mathrm{~s}$ at 1 -min intervals. PL vesicles were stored at $4^{\circ} \mathrm{C}$ and used within 1 month.

FVa-inactivation assays. FVa-inactivation studies using purified proteins involved two steps, an FVa-inactivation step and a step to quantitate residual FVa activity using either prothrombinase assays or coagulation assays.

Prothrombinase assays were performed with slight modification of protocols described previously (32). Aliquots containing FVa were withdrawn from reaction mixtures, and prothrombin activation was initiated by addition of factor $\mathrm{Xa}(1 \mathrm{nM}$ final), prothrombin ( $1 \mu \mathrm{M}$ final), and PL vesicles (20\% PE/5\% PS/75\% PC) ( $25 \mu \mathrm{M}$ final). At various times, aliquots were withdrawn and quenched with EDTA, and then thrombin amidolytic activity was quantitated (32). Thrombin amidolytic activity was linearly proportional to FVa between 1 and 20 pM FVa.

To study the time course of FVa inactivation, mixtures containing lipoproteins (equivalent to $200 \mu \mathrm{g} \mathrm{PL} / \mathrm{ml}$ ) or control buffer, $1.15 \mathrm{nM} \mathrm{APC}$, and $300 \mathrm{pM}$ FVa were incubated for 30 min at $37^{\circ} \mathrm{C}$ in the absence or presence of $14.5 \mathrm{nM}$ protein $\mathrm{S}$ or control buffer. Aliquots were withdrawn at $0,5,15,30$, and 60 min after the addition of FVa, and residual FVa clotting activity was determined in prothrombin-time assays using $50 \mu \mathrm{l}$ of FVdeficient plasma, $50 \mu \mathrm{l}$ of diluted test sample, and $50 \mu \mathrm{l}$ of Innovin. FVa at 0 min was measured using an aliquot withdrawn immediately after addition of FVa. Residual FVa activity was calculated from standard log-log calibration curves of clotting time versus [FVa] generated using purified $\mathrm{FVa}$.

For protein $\mathrm{S}$ dose-response studies that used FXa-1-stage clotting assays to quantitate $\mathrm{FVa}, \mathrm{FVa}(300 \mathrm{pM})$ was incubated with lipoproteins (equivalent to $270 \mu \mathrm{M} \mathrm{PL}), \mathrm{APC}(1.44 \mathrm{nM})$, and various concentrations of protein $\mathrm{S}$ for $30 \mathrm{~min}$ at $37^{\circ} \mathrm{C}$. Then $50 \mu \mathrm{l}$ aliquots were withdrawn, added to $50 \mu \mathrm{l}$ of FV-deficient plasma, and after $60 \mathrm{~s}$ at $37^{\circ} \mathrm{C}$, clotting was initiated by addition of $50 \mu \mathrm{l}$ containing $\mathrm{FXa}, \mathrm{CaCl}_{2}$, and PL vesicles (20\% $\mathrm{PE} / 5 \% \mathrm{PS} / 75 \% \mathrm{PC})(1.15 \mathrm{nM}, 8.3 \mathrm{mM}$, and $35 \mu \mathrm{M}$ final concentrations, respectively). FVa activity was calculated using standard curves prepared using purified FVa.

Immunoblot analysis. Aliquots taken at various times from FVainactivation reaction mixtures were added to hot SDS solution, subsequently electrophoresed on 4\%-15\% gradient SDS-polyacrylamide gels, and transferred to an Immobilon membrane (Millipore Corp., Bedford, Massachusetts, USA) as described (30). After being blocked with $1 \%$ casein in TBS, the membrane was incubated for $1.5 \mathrm{~h}$ at room temperature with the mixture of rabbit polyclonal and murine monoclonal antibodies against the FVa heavy chain. After washing, membranes were incubated with a mixture of biotinylated anti-rabbit IgG $(1 \mu \mathrm{g} / \mathrm{ml})$ (Pierce Chemical Co., Rockford, Illinois, USA) and biotinylated antimouse IgG $(1 \mu \mathrm{g} / \mathrm{ml}$ ) (Pierce Chemical Co.) for $1 \mathrm{~h}$, followed by streptavidin alkaline phosphatase $(1 \mu \mathrm{g} / \mathrm{ml})$ (Pierce Chemical Co.) for $45 \mathrm{~min}$. Finally, a nitroblue tetrazolium/5-bromo-4chloro-3-indolyl phosphate (NBT/BCIP) substrate kit (Bio-Rad

\section{Figure 1}

Influence of purified HDL and LDL on inactivation of purified normal Arg506-FVa or variant Gln506-FVa by purified APC and protein S. APC was incubated 30 min at $37^{\circ} \mathrm{C}$ with FVa (20 pM), HDL (260 $\mu \mathrm{M}$ PL; squares), LDL (260 $\mu \mathrm{M}$ PL; circles), or control buffer lacking lipoproteins (triangles) in either the absence ( $\boldsymbol{a}$ and $\boldsymbol{b}$ ) or presence ( $\boldsymbol{c}$ and d) of $14.5 \mathrm{nM}$ protein S. Residual FVa was determined using prothrombinase assays. FVa activity from controls without APC was defined as $100 \%$. HDL, high-density lipoprotein; $L D L$, low-density lipoprotein; $F V$ a, factor $V a$; $A P C$, activated protein $C ; P L$, phospholipid.

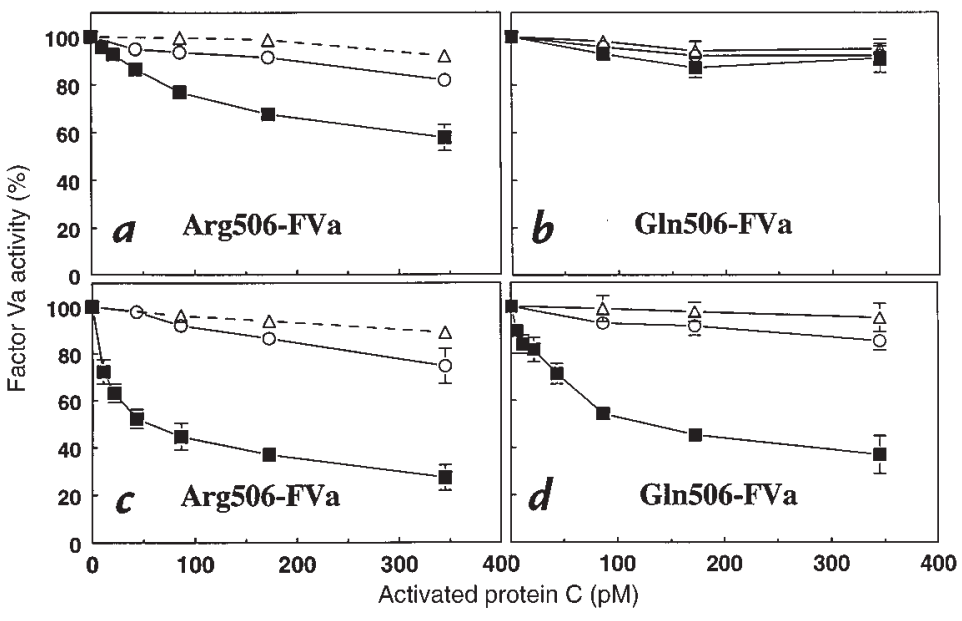


Laboratories Inc., Hercules, California, USA) was used for detection of FVa heavy-chain fragments.

Immunoadsorption analysis. Murine monoclonal IgG antibodies (MAB) (2 mg) against apoA-I designated AI-11 (28), apoB-100 designated MB20 (33), or glycoprotein IIbIIIa designated CP8 (34) (provided by Z. Ruggeri, The Scripps Research Institute) were coupled to $500 \mu \mathrm{l}$ of $\mathrm{CNBr}$-activated Sepharose following manufacturer's instructions (Pharmacia Biotech Inc., Piscataway, New Jersey, USA). Control CNBr-activated Sepharose without antibody addition was prepared using the same procedures. Coupling of each $\mathrm{MAB}$ was $>95 \%$. To test for immunoadsorption of HDL anticoagulant activity, $1 \mathrm{ml}$ of fresh (7 days old) HDL preparation $(560 \mu \mathrm{M} \mathrm{PL})$ was incubated overnight at $8^{\circ} \mathrm{C}$ with $500 \mu \mathrm{l}$ each of Sepharose-adsorbed MABs or control Sepharose beads, then separated by centrifugation. The anticoagulant cofactor activity of each supernatant was tested using purified Gln506-FVa as follows: $50 \mu \mathrm{l}$ of adsorbed solution was preincubated for varying times with $\mathrm{Gln} 506-\mathrm{FVa}(0.15 \mathrm{nM}$ final), protein $\mathrm{S}\left(14.5 \mathrm{nM}\right.$ final), and APC $\left(1.71\right.$ or $0.85 \mathrm{nM}$ final) at $37^{\circ} \mathrm{C}$. Aliquots were withdrawn and added to FV-deficient plasma to quantitate residual Gln506-FVa in FXa-1-stage assays. To define a standard curve for anticoagulant cofactor activity of adsorbed HDL supernatants, unadsorbed diluted HDL (400, 100, 25, and $0 \mu \mathrm{g} / \mathrm{ml} \mathrm{PL}$ ) was assayed for ability to enhance the rate of inactivation of Gln506-FVa using conditions given above and $5.1 \mathrm{nM}$ APC. A standard curve was constructed based on the relationship between HDL concentration and initial rate of inactivation of Gln506-FVa. The amount of residual anticoagulant cofactor activity of each adsorbed HDL preparation was quantitated based on this standard curve. To quantitate the amount of apoAI protein adsorbed by the $\mathrm{AI}-11 \mathrm{MAB} /$ Sepharose, $4 \%-15 \%$ gradient SDS-PAGE was used to analyze $10 \mu \mathrm{l}$ of each of the adsorbed fractions. Protein bands were stained with ISS Pro-Blue stain (Integrated Separation System, Natick, Massachusetts, USA), and the band intensity of apoA-I was quantitated by scans using an Eagle Eye II (Stratagene, La Jolla, California, USA) (courtesy of D. Salomon, The Scripps Research Institute).

Anticoagulant cofactor clotting assay. To determine anticoagulant or procoagulant activities of HDL, LDL, or PL vesicles, prothrombin-time assays were performed using an Amelung KC 4A micro apparatus (Sigma Diagnostics, St. Louis, Missouri, USA); $7.5 \mu \mathrm{l}$ normal plasma was mixed with $25 \mu \mathrm{l}$ containing fibrinogen $(4 \mathrm{mg} / \mathrm{ml})$ and APC $(96 \mathrm{nM})$ or buffer (TBS/2\% BSA), $50 \mu \mathrm{l}$ of HDL, LDL, PL vesicles, or buffer, and $25 \mu \mathrm{l}$ of protein S (420 $\mathrm{nM}$ ) or buffer, and incubated $3 \mathrm{~min}$ at $37^{\circ} \mathrm{C}$. Clotting times were measured after addition of $50 \mu \mathrm{l}$ containing 1:64 or 1:40 dilution of recombinant human tissue factor (Innovin; BaxterDade), $30 \mathrm{mM} \mathrm{CaCl}_{2}$, and TBS/2\% BSA. For studies of the anticoagulant response to APC/protein S of 20 normal plasmas, the assay was similarly performed except that no HDL or LDL was added and a 1:32 dilution of Innovin was used. The same normal plasma samples were also assayed using the activated partial thromboplastin time (APTT)-based APC resistance test (35) with Platelin LS (36). The APC resistance ratio was calculated by dividing the APTT in the presence of added APC by the baseline APTT. Although the term APC sensitivity seems more logical to describe this ratio, the term APC resistance follows historical usage and herein is restricted to APTT-based tests.

Statistical analysis. Linear regression and correlation analyses were made using GraphPad Prism (version 2.0) software (GraphPad Software for Science Inc., San Diego, California, USA). Pearson correlation coefficients are reported, and statistical significance was achieved when two-tailed $P$ values were $<0.05$.

\section{Results}

Influence of HDL or LDL on APC anticoagulant activity. To determine the influence of purified HDL and LDL frac-

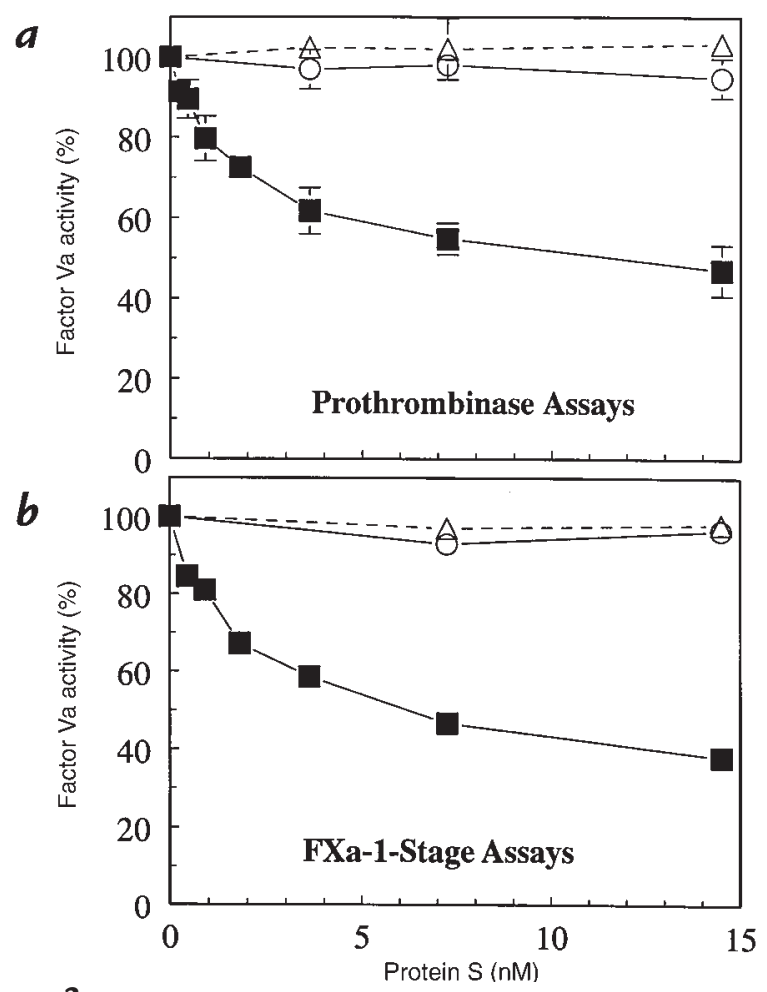

Figure 2

Influence of protein $\mathrm{S}$ on inactivation of FVa by APC in the presence or absence of HDL and LDL. FVa $(20 \mathrm{pM})$ was incubated for 30 min with 350 PM APC and varying amounts of protein $\mathrm{S}$ in the presence of $\mathrm{HDL}(260 \mu \mathrm{M}$ $\mathrm{PL}$; squares), LDL (260 $\mu \mathrm{M} \mathrm{PL}$; circles), or control buffer (triangles). Residual FVa was quantified using either $(\boldsymbol{a})$ prothrombinase assays or ( $\boldsymbol{b}$ ) FXa-1-stage clotting assays. FVa activity observed for APC alone was defined as $100 \%$.

tions on anticoagulant activities of the protein $\mathrm{C}$ pathway, inactivation of purified coagulation FVa by APC and its cofactor, protein $\mathrm{S}$, was studied using a two-step procedure. First, FVa was incubated with LDL, HDL or buffer, $\mathrm{APC}$, and protein $\mathrm{S}$ to inactivate FVa. Second, residual FVa activity was quantitated using either prothrombinase assays or coagulation assays that employed FV-deficient plasma. The effects of purified lipoproteins on the APC dose-responses for inactivation of normal Arg506-FVa and of the variant Gln506-FVa are seen in Fig. 1. For normal Arg506-FVa, data (Fig. 1, $a$ and $c$ ) show that HDL but not LDL markedly enhanced APC-dependent loss of FVa activity and that this anticoagulant effect of HDL was more pronounced in the presence of protein $S$.

To clarify the importance of HDL for cleavage at Arg306, experiments using purified Gln506-FVa variant were compared with those using Arg506-FVa (Fig. 1). Inactivation of the FVa variant is due to specific cleavage at Arg306 because APC does not cleave at $\operatorname{Gln} 506$ (25, 30, 37). Without protein S (Fig. $1 b$ ), no inactivation of Gln506-FVa by APC was seen in the absence or presence of HDL or LDL. However, in the presence of protein S, HDL-dependent inactivation of Gln506-FVa by APC was observed (Fig. 1d). These results suggest that protein $S$, along with $\mathrm{HDL}$, is responsible for proteolytic inactivation of FVa because of cleavage at Arg306 by APC (vide infra).

Influence of $H D L$ or $L D L$ on protein S APC-cofactor activity. To study the effect of HDL and LDL on protein S APC- 


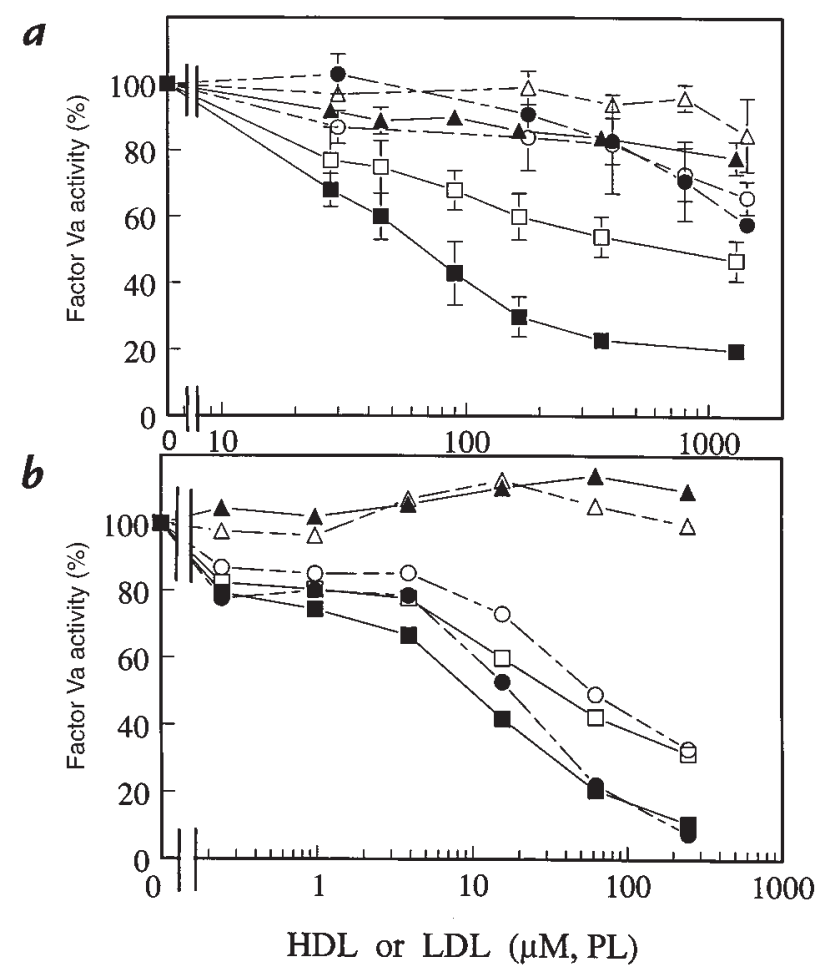

Figure 3

Influence of purified HDL and LDL or of their PL extracts on inactivation of FVa by APC and protein S. (a) HDL (squares) or LDL (circles) were incubated with $\mathrm{FVa}(20 \mathrm{pM}$ ) for 30 min with $350 \mathrm{pM}$ APC alone (open symbols), $350 \mathrm{pM} \mathrm{APC}$ and $14.5 \mathrm{nM}$ protein S (solid symbols), or control buffer (HDL, solid triangles; LDL, open triangles). Residual FVa was measured using prothrombinase assays. FVa activity without HDL or LDL was defined as $100 \%$ activity. (b) PL extracts from HDL (squares) or LDL (circles) were tested for their ability to enhance $\mathrm{FVa}$ inactivation using the same reagents and conditions as in $a$. Symbols represent the same variables (APC alone, APC plus protein $\mathrm{S}$, or control buffer) as in $a$. Concentrations of LDL and HDL or their PL extracts are given based on content of choline-containing PL.

cofactor activity, FVa was incubated with a low level of $\mathrm{APC}$ and lipoproteins or buffer at varying protein S concentrations for $30 \mathrm{~min}$, and then residual FVa was quantitated using prothrombinase assays (Fig. $2 a$ ) or FXa1 -stage clotting assays (Fig. 2b). HDL, but not LDL, enhanced the anticoagulant activity of protein $S$, and the quantitation of loss of FVa activity was essentially the same based on either prothrombinase assays (Fig. $2 a$ ) or FXa-1-stage clotting assays (Fig. 2b). Under the conditions employed, no FVa inactivation was observed in the presence of LDL or in the absence of lipoproteins (Fig. 2) or APC (data not shown). Under the conditions employed, $7.3 \mathrm{nM}$ protein $\mathrm{S}$ was required for $\sim 50 \%$ inhibition of FVa by APC. Free protein S, which exhibits APC-cofactor activity, is present in normal human plas$\mathrm{ma}$ at $145 \mathrm{nM}$ (38), indicating that the protein S effects could occur within its physiological range.

Dose dependence for anticoagulant activity of $H D L$ or $L D L$ or their PL extracts. To define the influence of HDL and LDL concentrations on FVa inactivation, experiments similar to those above were performed (Fig. 3a). HDL at or below plasma levels showed potent anticoagulant cofactor activity (i.e., loss of FVa activity) when either APC alone or APC plus protein S were present (Fig. $3 a$, lower two curves). For example, HDL at $0.4 \mathrm{mM}$ PL equivalent supported $45 \%$ and $80 \%$ loss of FVa activity by APC alone and APC plus protein S, respectively (Fig. $3 a$ ). In the absence of lipoproteins, no measurable FVa inactivation occurred under the conditions employed. In the presence of LDL, APC in the presence or absence of protein S caused at most only modest inactivation of FVa, and HDL was clearly much more active than LDL. For example, in the presence of APC and protein S, 40\% loss of FVa activity was observed at $43 \mu \mathrm{M} \mathrm{PL} / \mathrm{HDL}$ and at $1.3 \mathrm{mM}$ $\mathrm{PL} / \mathrm{LDL}$, suggesting that HDL was $\sim 30$ times more active than LDL when compared on the basis of choline-containing PL content. The highest HDL and LDL concentrations studied in Fig. $3 a$ correspond to $\sim 135 \%$ of normal plasma levels of HDL or LDL, i.e., equivalent to 1.3 $\mathrm{mM}$ choline-containing PL versus the normal plasma values equivalent to $0.94 \mathrm{mM}$ choline-containing PL. Thus, these results show that HDL at levels present in normal plasma can support significant APC anticoagulant activity in the presence of protein $S$.

PL extracts were prepared from purified LDL and HDL and tested in dose-response studies for their ability to enhance FVa inactivation by APC and protein S (Fig. 3b). In the presence of APC and protein S, 50\% inactivation of FVa required $10 \mu \mathrm{M}$ of HD-PL extract or $15 \mu \mathrm{M}$ LDL-PL extract, respectively (Fig. 3b). This can be compared with $70 \mu \mathrm{M}$ of $\mathrm{HDL}$ or $>1.5 \mathrm{mM} \mathrm{LDL}$ required for $50 \% \mathrm{FVa}$ inactivation (Fig. $3 a$ ), suggesting that purified HDL is about $15 \%$ as active as its component PL extract, whereas LDL is less than $1 \%$ as active as its component PL extract. Influence of HDL and LDL on FVa cleavage by APC and protein $S$. During proteolytic inactivation, APC cleaves the FVa heavy chain at Arg506, Arg306, and perhaps Arg679; cleavage at Arg306 is critical for complete inactivation of FVa or FV (25). Protein S and PL vesicles each enhance more than 10-fold Arg306 cleavage, whereas protein S does not markedly enhance Arg506 cleavage $(25,26)$. Immunoblotting studies performed using anti-FVa heavy-chain antibodies (Fig. 4) showed that APC/protein $\mathrm{S}$ caused a time-dependent appearance of $78-\mathrm{kDa}$ bands, representing the residues $1-506$ fragment $(25,26,30)$ in reaction mixtures containing $\mathrm{HDL}$, LDL, or control buffer. Thus, neither HDL nor LDL appeared to markedly affect the rate of cleavage of FVa by APC at Arg506. However, only in the presence of HDL was there a timedependent appearance of 44- and 30-kDa bands (Fig. 4) representing bands typical of the residues 1-306, 307-506, and 507-709 fragments, respectively $(25,26$, 30). In parallel studies in the presence of APC but absence of protein $\mathrm{S}$, immunoblots showed a time-dependent appearance of the 78-kDa band but no smaller 44- and 30-kDa bands (data not shown), indicating that under these experimental conditions protein $\mathrm{S}$ was required for cleavage at or near Arg306. In the absence of APC, no FVa fragments were generated (data not shown).

Requirement for phospholipids and apoA-I in HDL. The enhancement of APC-dependent anticoagulant activity by HDL seen above resembles the action of anionic PLs that enhance protein S-dependent cleavage at Arg306 in FVa by APC (26). To assess whether PL in HDL was necessary for its anticoagulant cofactor activity, HDL (500 
$\mu \mathrm{M}$ PL) was pretreated for 15 minutes at $37^{\circ} \mathrm{C}$ with purified phospholipase $\mathrm{A}_{2}$ from either bee venom (1.8 nM) or Naja mossambica mossambica venom $(0.45 \mathrm{nM})$ before addition of APC (0-340 pM) and FVa (20 pM) in the absence or presence of protein $\mathrm{S}(29 \mathrm{nM})$. After $30 \mathrm{~min}$ utes, residual $\mathrm{FVa}$ was quantitated essentially as described (Fig. 1). In results not shown, each phospholipase $\mathrm{A}_{2}$ ablated the anticoagulant cofactor activity of HDL, suggesting that intact PL in HDL was necessary.

To define the active molecular species in HDL, immobilized antibodies against apoA-I, apoB-100, or glycoprotein IIbIIIa were tested for their ability to adsorb anticoagulant activity. Anti-apoA-I IgG adsorbed 80\% of the anticoagulant activity, whereas no significant $(<8 \%)$ adsorption was observed for the other antibodies or control Sepharose beads (data not shown). Based on sodium dodecyl sulfate-polyacrylamide gel electrophoresis (SDS-PAGE) and densitometric analyses, immobilized anti-apoA-I antibody adsorbed the target apoA-I antigen; i.e., the stained apoA-I protein band seen for the supernatant of HDL samples adsorbed with anti-apoA-I/Sepharose was $14 \%$ of the band seen for parallel HDL samples adsorbed with control Sepharose beads or with Sepharose beads containing immobilized MABs against apoB-100 or glycoprotein IIbIIIa. Thus, HDL containing apoA-I provided the anticoagulant activity observed, and anticoagulant activity was not due to contamination of HDL with glycoprotein IIbIIIa-bearing platelet microparticles.

$H D L$ anticoagulant activity in clotting assays of normal plasma. To demonstrate the anticoagulant cofactor activity of HDL in the plasma milieu, a modified prothrombintime clotting assay was developed. As seen in Fig. 5, in the presence of APC and protein S, addition of purified HDL caused dose-dependent marked prolongation of this modified prothrombin-time assay whereas LDL had only a slight effect. For example, when HDL in the clotting assay mixture was increased from $\sim 5 \%$ to $40 \%$ of its normal plasma level $(0.94 \mathrm{mM})$, a 300 -second prolongation was observed compared with a 28 -second prolongation by LDL (Fig. 5). In the absence of added APC and protein S, neither HDL nor LDL had any effect on the clotting time (Fig. 5). When APC alone was added without protein $\mathrm{S}$, addition of the lipoproteins caused a slight prolongation of the clotting times. When protein $S$ was present in the absence of added APC, neither HDL nor LDL had any effect on the clotting times (data not shown). When the anticoagulant activity of HDL prepared from six unrelated blood donors was assayed in HDL dose-response studies as in Fig. 5, similar results were obtained (data not shown). Based on the HDL dose-response curves, the variation in HDL specific activity was $<40 \%$ among the six donors' HDL preparations (data not shown). Thus, HDL purified from six different donors showed similar significant anticoagulant cofactor activity when APC and protein S were added.

Experiments were performed to characterize the anticoagulant potency of HDL compared with PL vesicles of welldefined composition. The dilute prothrombin time was determined in the absence and presence of APC and protein $\mathrm{S}$ at varying concentrations of $\mathrm{PL}$ vesicles containing either $20 \%$ PS/80\% PC or $1 \%$ PS/3\% PE/96\% PC, the former being optimal for PL-dependent coagulation reactions and the latter containing the known PS and PE content of HDL. The results in Fig. 6 show that the PL vesicles appeared procoagulant only below $25 \mu \mathrm{M}$ and above 25 $\mu \mathrm{M}$ appeared anticoagulant in the absence or presence of $\mathrm{APC}$ and protein $\mathrm{S}$. The ratio of the clotting time in the presence of added APC and protein $S$ to that observed in their absence reflects the anticoagulant response to $\mathrm{APC} /$ protein S. When ratios for PL vesicles were compared with those for HDL (Fig. 6, inset), it appeared that HDL was qualitatively different from the two PL vesicles because HDL showed good anticoagulant activity but no procoagulant activity, whereas the PL vesicles were essentially rather procoagulant and gave a ratio <3.0, the ratio observed for no PL or HDL additions. On net balance, the anticoagulant activity of HDL added to plasma was much greater than the activity of these vesicles; e.g., at $400 \mu \mathrm{M}$ PL, the ratio was $>5$ for HDL but only 2.0-2.1 for PL vesicles (less than control with no added PL). Moreover, it was clear that HDL up to $1,000 \mu \mathrm{M}$ PL exhibited no procoagulant activity in the absence of APC and protein S, whereas vesicles with the $1 \%$ PS $/ 3 \%$ PE content of HDL, which shortened the clotting time from 52 seconds to 40 seconds at $400 \mu \mathrm{M}$ PL (Fig. 6), were procoagulant.

To assess the correlation between endogenous HDL plasma levels and anticoagulant response to APC/pro-

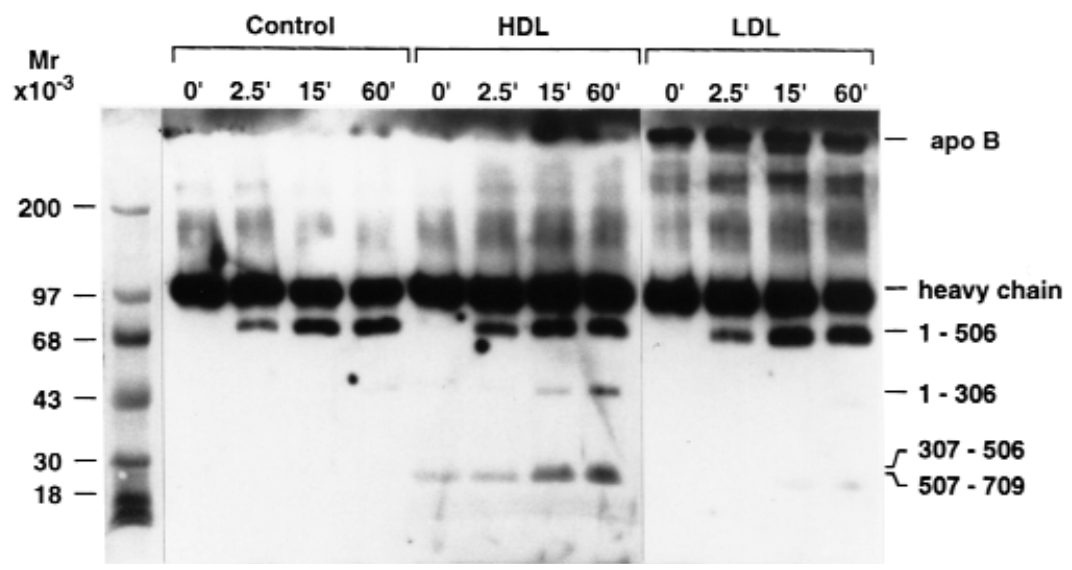

Figure 4

Immunoblot analysis of cleavage of normal FVa by $\mathrm{APC} /$ protein $\mathrm{S}$ in the presence of purified $\mathrm{HDL}$ or LDL. Purified human FVa $(18 \mathrm{nM})$ was incubated with APC (4.3 nM), $\mathrm{CaCl}_{2}(5 \mathrm{mM}), 0.1 \% \mathrm{BSA}$, and protein $\mathrm{S}(58 \mathrm{nM})$ in the absence of lipoproteins (control lanes 2-5) or in the presence of HDL (260 $\mu \mathrm{M}$ PL) (lanes 6-9) or LDL (260 $\mu \mathrm{M} \mathrm{PL}$ ) (lanes 10-13). At the times indicated, aliquots were taken and analyzed by immunoblotting using anti-FVa heavy-chain antibodies. 
tein S, fasting plasma samples from 20 healthy adults were tested using the modified prothrombin-time assay. The level of apoA-I antigen was determined as an indicator of HDL protein in each plasma. The anticoagulant response to added $\mathrm{APC} /$ protein $\mathrm{S}$ was correlated with the level of apoA-I $(r=0.47, P=0.035)$ (Fig. $7 a)$. However, given the obvious variability and scatter in these data, a number of other plasma factors contribute to the variability in anticoagulant response in APC/protein S. The standard APTT-based APC resistance test was also performed on parallel plasma samples from the same 20 blood donations. In contrast to the prothrombin time-based test results (Fig. 7a), there was no statistically significant correlation between the APTT-based APC resistance ratio and plasma apoA-I levels $(r=-0.12, P=$ 0.63) (Fig. 7b). Moreover, comparison of the prothrombin time-based APC/protein S sensitivity test results with the APTT-based APC resistance ratio showed no statistically significant correlation $(r=0.25, P=0.26)$.

\section{Discussion}

Experiments reported here demonstrate for the first time that HDL, a generally beneficial heterogeneous fraction of plasma lipoproteins, can significantly enhance anticoagulant activity exerted by APC and protein S. Studies using purified lipoproteins and coagulation factors show that HDL but not LDL enhances APC inactivation of FVa as well as protein S cofactor activity for this reaction. Results for quantitation of FVa inactivation obtained using clotting assays were consistent with results from prothrombinase assays. When different preparations of protein S or HDL from different healthy adult donors were used, the same results were observed. Thus, the conclusion that HDL is an anticoagulant cofactor in purified reaction mixtures was not dependent on either the method used to

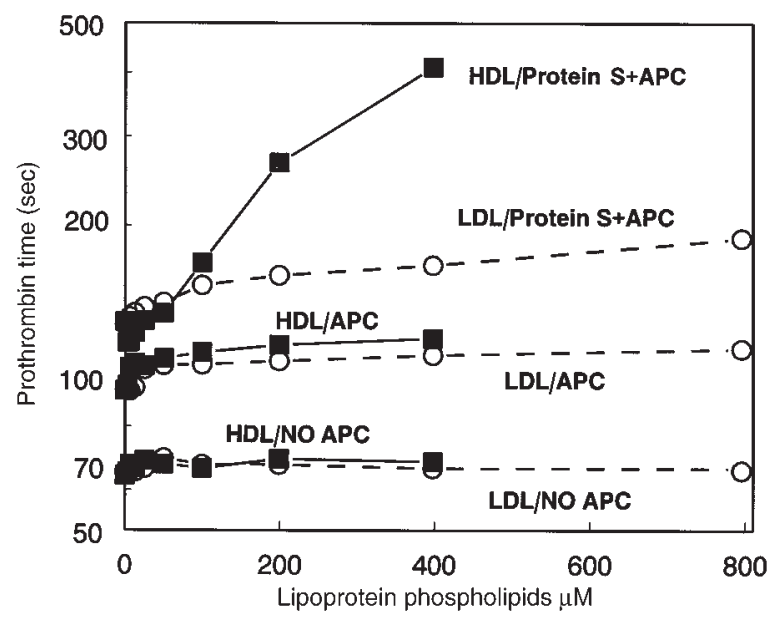

Figure 5

Anticoagulant cofactor activity of HDL in clotting assays in the presence of APC/protein S. APC and protein S, APC alone, or control buffer without $A P C$ protein $S$ (as indicated) were mixed with plasma, fibrinogen, and purified lipoproteins, and clotting was initiated by addition of tissue factor $/ \mathrm{Ca}^{++}$(see Methods). Final LDL (circles) or HDL (squares) concentration was based on choline-containing PL content. quantitate FVa inactivation or the donor of purified reagent lipoproteins or clotting factors. The anticoagulant cofactor activity of HDL preparations is indeed due to the HDL particles, because anti-apoA-I antibodies adsorbed the activity while anti-glycoprotein IIbIIIa and anti-apoB-100 antibodies had no effect.

Dose-dependent anticoagulant activity of purified HDL is demonstrable in the plasma milieu in the presence, but not the absence, of APC and protein S when dilute tissue factor is used to initiate clotting (Fig. 5). Moreover, the anticoagulant response to added $\mathrm{APC} /$ protein $\mathrm{S}$ in our modified prothrombin-time assay is correlated with the level of apoA-I (Fig. 7a), the major protein of HDL. The latter observation supports the conclusion that apoA-I bearing HDL particles are responsible for the anticoagulant cofactor activity of purified HDL. The coefficient of determination for apoA-I and anticoagulant response to APC/protein $S$ is 0.22 , suggesting that, at least in vitro, plasma HDL levels may exert a significant influence on the anticoagulant response of the protein $C$ pathway.

The dilute prothrombin-time assay in which both the tissue factor reagent and the test plasma are diluted and to which we add fibrinogen was developed to be sensitive to endogenous plasma lipids and lipoproteins in its response to APC/protein S. Standard APTT assays involve addition of PL reagents that tend to mask the procoagulant and anticoagulant properties of endogenous plasma lipids. Furthermore, the standard APTT-based APC resistance test is not sensitive to added protein $S$ (39), whereas protein $\mathrm{S}$ addition permits marked HDL influence on our prothrombin time-based APC sensitivity assay (Fig. 5). Interestingly, the traditional APTT-based APC resistance test results are not significantly correlated either with apoA-I levels (Fig. 7b) or with the response to APC/protein S measured using the dilute prothrombin-time clotting assay. Therefore, published information about risks of thrombotic disease associated with "APC resistance" that was diagnosed using APTT-based assays involving either neat patient plasma (35) or patient plasma diluted into FV-deficient plasma (40) cannot simply be extrapolated to the relationship between prevalence or risk of thrombotic diseases and sensitivity to APC/protein $\mathrm{S}$ diagnosed using our prothrombin time-based assay. In general terms, the in vitro anticoagulant response to APC in different clotting assays may be more or less sensitive to plasma components, depending on the design of the clotting assay. Hence, the terms APC resistance or APC sensitivity should be carefully qualified in the context of the experimental conditions.

As a corollary to the proposed relationship between HDL and the anticoagulant protein $\mathrm{C}$ pathway, one might speculate that protein $\mathrm{C}$ or protein $\mathrm{S}$ deficiency would predispose a patient to atherosclerosis. No adult with severe homozygous deficiency for either of these autosomal traits has been identified. The majority of surviving homozygous protein $\mathrm{C}$-deficient children are quite young and do not provide informative data regarding this speculation. Although reports of arterial thrombosis associated with protein $\mathrm{C}$ and protein $\mathrm{S}$ heterozygous deficiency have appeared (41-43), it has not been established that heterozygous deficiency of these pro- 


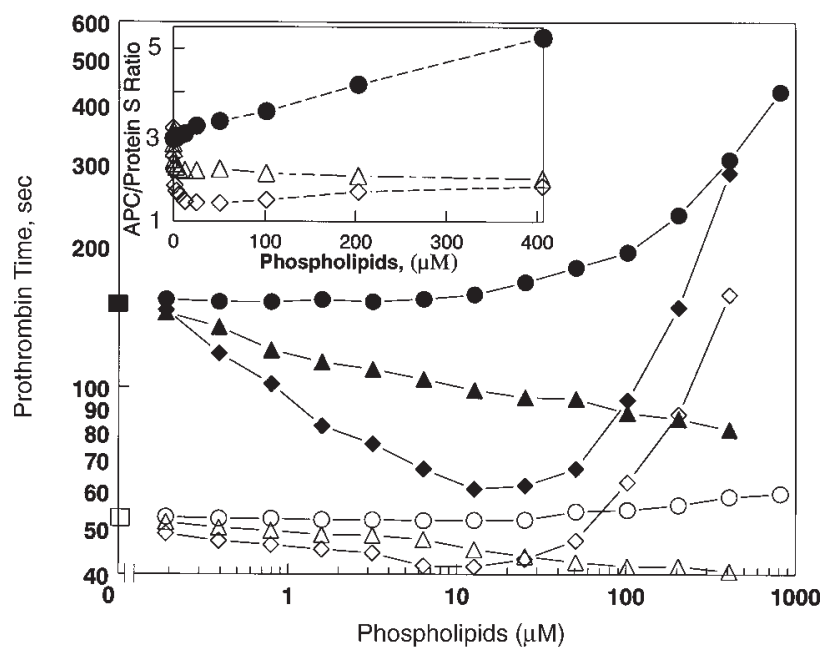

teins is a predisposing risk factor. Although one might look for an inverse correlation between zymogen protein $\mathrm{C}$ antigen level and incidence of coronary heart disease, no such relationship was found in the Atherosclerosis Risk in Communities (ARIC) study (44). However, we note that HDL modulates the anticoagulant response to $\mathrm{APC} /$ protein $\mathrm{S}$ and that this response does not depend on the level of protein $\mathrm{C}$ zymogen. Interestingly, the relative risk of ischemic stroke in the ARIC study was inversely correlated with protein C levels (45), suggesting that protein $\mathrm{C}$ is neuroprotective for cerebral arterial thrombosis. New appropriately designed clinical and animal model studies are needed to assess the hypothesis that HDL may modulate the expression of protein C pathway activities, especially in the brain. One might also ask whether high HDL is protective with respect to venous thrombosis; we are unaware of any useful study of this question. Hyperlipidemia is apparently a risk factor for venous thrombosis (46). One should note that mechanisms for pathophysiology of arterial disease, including arterial thrombosis, only partially overlap those for venous disease and venous thrombosis.

Limited proteolysis of the FVa heavy chain by APC was studied using immunoblot analysis to clarify the mechanisms by which HDL contributes to APC anticoagulant activity. APC inactivates FVa by cleavage first at Arg506, then at Arg306 (25), the latter but not the former cleavage being greatly enhanced by protein $S(26)$. Overall, the results from functional assays (Fig. 1) and immunoblot studies (Fig. 4) are consistent with the hypothesis that interactions involving both protein $\mathrm{S}$ and HDL contribute to APC activity by promoting FVa cleavage specifically at Arg306.

When the net procoagulant and/or anticoagulant activity of HDL added to plasma was compared with PL vesicles (Fig. 6), synthetic PL vesicles containing either 20\% $\mathrm{PS} / 80 \% \mathrm{PC}$ or $1 \% \mathrm{PS} / 3 \% \mathrm{PE} / 96 \% \mathrm{PC}$ were procoagulant, whereas HDL was anticoagulant. Thus, there are both qualitative and quantitative differences between HDL and PL vesicles in these clotting assays. Thus, HDL does not simply provide a PL surface as do PL vesicles, and HDL appears to have unique anticoagulant cofactor properties. Because phospholipase $\mathrm{A}_{2}$ abrogates the activity, $\mathrm{PL}$ is

\section{Figure 6}

Comparison of HDL anticoagulant potency with PL vesicles. The modified prothrombin-time assay was performed in the absence (open symbols) and presence (solid symbols) of added APC/protein S (see Methods) and varying concentrations of HDL (circles), PL vesicles containing 20\% PS/80\% PC (diamonds), or 1\% PS/3\% PE/96\% PC (triangles). Squares indicate absence of added HDL or PL. Inset shows the ratio of the clotting time in the presence of added APC/protein S to that without added APC/protein S.

essential for HDL's anticoagulant action. When PL extracts of purified HDL and LDL were normalized for choline-containing PL and compared for their ability to support APC and protein S action, they were similar, with HDL-PL being 1.5-fold more active than LDL-PL (Fig. 3b). However, when HDL and LDL were similarly compared, HDL was $\sim 30$ times more active than LDL (Fig. $3 a$ ). HDL had $\sim 15 \%$ the activity of its PL extract, whereas LDL had $<1 \%$ of its PL activity, suggesting that incorporation of PL into these lipoproteins markedly restricts the availability of PL to support coagulation reactions. Because the overall PL content and composition of HDL particles are not significantly different from those of LDL, it is unclear why HDL but not LDL particles provide a supporting surface for the action of protein S and APC. The anticoagulant effect of HDL may result from high affinity of protein $S$ for HDL particles due to some unique structural HDL properties, such as size of particles and content of lipids and/or PLs, and/or qualitative differences, such as net charge or polarity. Alternatively or additionally, the apolipoproteins of HDL may organize PLs into domains that favor interaction with protein S, APC, and/or FVa. HDL's apolipoproteins may interact specifically with APC, protein S, or FVa. The chemical heterogeneity of HDL particles underlies their multiple and variable functional properties.

In vitro studies have shown that APC-dependent anticoagulant activity in the presence of protein $S$ is significantly enhanced by PE (47) or cardiolipin (Fernández, J.A., et al., unpublished data) when either is incorporated into multicomponent PL vesicles. It was suggested that these two PLs are more anticoagulant than procoagulant, at least in the presence of APC and protein S, suggesting that PL composition of vesicles may alter their hemostatic properties. In extending this concept to HDL particles, we speculate that $\mathrm{PE}$ and/or cardiolipin as anticoagulant PLs may contribute to the anticoagulant APC/protein S cofactor activity of HDL. It would follow that HDL subfractions enriched in PE and/or cardiolipin may possess a higher specific activity as an anticoagulant cofactor. Patients with CAD have lower HDL-PL levels $(48,49)$, and decreases in HDL-PL levels were reported to correlate better with CAD than with the decrease in HDL-cholesterol 


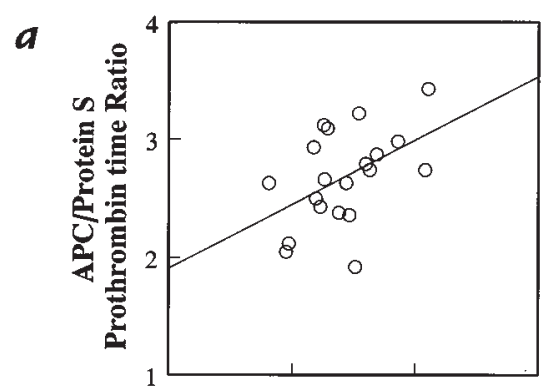

b

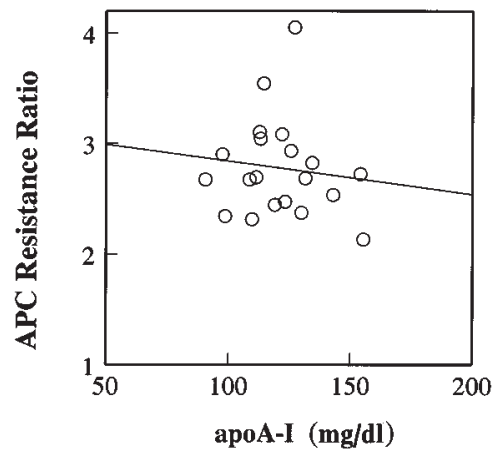

\section{Figure 7}

Correlation between plasma apoA-I levels and anticoagulant response to either $(\boldsymbol{a}) \mathrm{APC}$ /protein $\mathrm{S}$ in prothrombin-time assays or $(\boldsymbol{b})$ APC in APTT assays. Levels of apoA-I antigen were measured in 20 normal healthy adult plasmas ( 11 females, 9 males) and analyzed for correlation with either (a) anticoagulant response to APC/protein S in dilute prothrombin-time assays or $(b)$ anticoagulant response to APC in APTT assays (35, 36). Anticoagulant response is reported as the ratio of the clotting time observed in the presence of added (a) APC/protein S or (b) APC alone to the respective control clotting time observed in the absence of added anticoagulant factors. Statistical analysis: $(a) r=0.47, P=0.035 ;(b) r=$ -0.12; $P=0.63$. APTT, activated partial thromboplastin time.

levels (50). Furthermore, HDL in plasma of subjects with high HDL levels has relatively higher levels of PE than HDL in subjects with normal HDL levels (51). These observations are consistent with a physiological role for HDL-PL in the regulation of atherothrombosis.

Based on the present study involving purified reagents, we propose that the atheroprotective activity of plasma HDL may be based, at least in part, on HDL's ability to enhance the anticoagulant action of protein S-dependent APC cleavage of FVa or FV at Arg306. Extensive clinical research, animal model studies, and laboratory investigations all support the proposition that the protein $\mathrm{C}$ pathway provides a major antithrombotic mechanism involving downregulation of thrombin generation by blood coagulation pathways. The inverse relationship between plasma HDL levels and CAD or acute arterial thrombotic events is widely recognized. This new hypothesis that HDL exerts anticoagulant activity via the protein C pathway connects these two broad observations, bridges these areas of investigation, and encourages basic and clinical studies to assess the significance of this hypothesis. Related to this concept, a recent report demonstrated that infusion of reconstituted HDL into human volunteers who had also received lipopolysaccharides provided remarkable in vivo protective effects, including an apparent anticoagulant effect (52). Given the complexity of plasma HDL molecular species, significant efforts are needed to identify the biochemical and structural properties of the subclasses of HDL that are most responsible for HDL's anticoagulant properties.

Plasma lipoproteins have procoagulant effects $(12,53$, 54). Certain steps in the blood coagulation pathways are greatly enhanced by anionic PL and vitamin K-dependent clotting factors, and FVa and FVIIIa bind to PL vesicles. A positive relationship between levels of blood coagulation factors and hyperlipidemic status was suggested because antigen and/or activity levels of vitamin $\mathrm{K}$-dependent coagulation factors are increased in patients with hyperlipidemia or in alimentary lipemia $(55,56)$. Plasma free fatty acids, especially those generated postprandially, may stimulate the contact activation system, generation of factor XIIa, kallikrein, and factor VIIa $(12,14,15)$, and related sequelae (57). Interactions between plasma lipoproteins and procoagulant factors were reported $(54,58-60)$. These various observations support the hypothesis that molecular interactions of clotting factors with plasma lipids and lipoproteins can promote procoagulant reactions and thrombin generation. The new hypothesis that HDL exerts APC/protein S-dependent anticoagulant activity supports a paradigm in which plasma lipids and lipoproteins provide a background on which the yin-yang patterns of procoagulant and anticoagulant potentials may be envisioned.

\section{Acknowledgments}

Helpful discussions with M.J. Heeb and Y. Kojima, who also provided Gln506-FVa, are gratefully acknowledged. This study was supported in part by National Institutes of Health grants RO1HL21544, R37HL52246, RO1HL43815, RO1HL50060, and MO1RR00833, and by the Stein Endowment Fund.

1. Gordon, D.J., et al. 1989. High-density lipoprotein cholesterol and cardiovascular disease. Four prospective American studies. Circulation. 79:8-15.

2. Barter, P.J., and Rye, K.-A. 1996. High density lipoproteins and coronary heart disease. Atherosclerosis. 121:1-12.

3. Tall, A.R. 1990. Plasma high density lipoproteins. Metabolism and relationship to atherogenesis. J. Clin. Invest. 86:379-384.

4. Tribble, D.L., and Krauss, R.M. 1993. HDL and coronary artery disease. Adv. Intern. Med. 38:1-29.

5. Miller, N.E., La Ville, A., and Crook, D. 1985. Direct evidence that reverse cholesterol transport is mediated by high-density lipoprotein in rabbit. Nature. 314:109-111.

6. Fielding, C.J., and Fielding, P.E. 1995. Molecular physiology of reverse cholesterol transport. J. Lipid Res. 36:211-228.

7. Parthasarathy, S., Barnett, J., and Fong, L.G. 1990. High-density lipoprotein inhibits the oxidative modification of low-density lipoprotein. Biochim. Biophys. Acta. 1044:275-283.

8. Banka, C.L. 1996. High density lipoprotein and lipoprotein oxidation. Curr. Opin. Lipidol. 7:139-142.

9. Berliner, J.A., et al. 1995. Atherosclerosis: basic mechanisms. Oxidation, inflammation, and genetics. Circulation. 91:2488-2496.

10. Barter, P.J., et al. 1998. Antiatherogenic effects of high-density lipoproteins: mechanisms. In Atherosclerosis XI. B. Jacotot, D. Mathé, and J.-C. Fruchart, editors. Elsevier Science. Singapore. 125-133.

11. Ross, R. 1993. The pathogenesis of atherosclerosis: a perspective for the 1990s. Nature. 362:801-809.

12. Miller, G.J. 1995. Lipoproteins and thrombosis: effects of lipid lowering. Curr. Opin. Lipidol. 6:38-42.

13. Miller, G.J., Bauer, K.A., Barzegar, S., Cooper, J.A., and Rosenberg, R.D. 1996. Increased activation of the haemostatic system in men at high risk of fatal coronary heart disease. Thromb. Haemost. 75:767-771.

14. Mitropoulos, K.A. 1994. Lipid-thrombosis interface. Br. Med.J. 50:813-832.

15. Hamsten, A., Karpe, F., Båvenholm, P., and Silveira, A. 1994. Interactions amongst insulin, lipoproteins and haemostatic function relevant to coronary heart disease. J. Intern. Med. 236:75-88.

16. Marlar, R.A., Kleiss, A.J., and Griffin, J.H. 1982. Mechanism of action of 
human activated protein $\mathrm{C}$, a thrombin-dependent anticoagulant enzyme. Blood. 59:1067-1072.

17. Esmon, C.T. 1993. Molecular events that control the protein C anticoagulant pathway. Thromb. Haemost. 70:29-35.

18. Dahlbäck, B. 1995. The protein C anticoagulant system: inherited defects as basis for venous thrombosis. Thromb. Res. 77:1-43.

19. Griffin, J.H., and Petaja, J. 1997. Activated protein C resistance. In Molecular mechanisms of hypercoaguable states. A.I. Schafer, editor. Landes Bioscience. Austin, TX. 101-123.

20. Fisher, M., et al. 1996. Activated protein C resistance in ischemic stroke not due to factor $\mathrm{V}$ arginine $\mathrm{a}^{506} \rightarrow$ glutamine mutation. Stroke. 27:1163-1166.

21. van der Bom, J.G., et al. 1996. Reduced response to activated protein C is associated with increased risk for cerebrovascular disease. Ann. Intern. Med. 125:265-269.

22. Ridker, P.M., et al. 1995. Mutation in the gene coding for coagulation fac tor $\mathrm{V}$ and the risk of myocardial infarction, stroke, and venous thrombosis in apparently healthy men. N. Engl. J. Med. 332:912-917.

23. Esmon, C.T. 1997. Defects in natural anticoagulant pathways as potential risk factors for myocardial infarction. Circulation. 96:9-11.

24. Bakker, H.M., et al. 1992. The effect of phospholipids, calcium ions and protein $\mathrm{S}$ on rate constants of human factor Va inactivation by activated human protein C. Eur. J. Biochem. 208:171-178

25. Kalafatis, M., Rand, M.D., and Mann, K.G. 1994. The mechanism of inactivation of human factor $\mathrm{V}$ and human factor Va by activated protein $\mathrm{C}$. J. Biol. Chem. 269:31869-31880.

26. Rosing, J., et al. 1995. Effects of protein S and factor Xa on peptide bond cleavages during inactivation of factor $\mathrm{Va}$ and factor $\mathrm{Va}^{\mathrm{R} 506 \mathrm{Q}}$ by activated protein C. J. Biol. Chem. 270:27852-27858.

27. Banka, C.L., Black, A.S., Dyer, C.A., and Curtiss, L.K. 1991. THP-1 cells form foam cells in response to coculture with lipoproteins but not platelets. J. Lipid Res. 32:35-43.

28. Banka, C.L., Bonnet, D.J., Black, A.S., Smith, R.S., and Curtiss, L.K. 1991. Localization of an apolipoprotein A-I epitope critical for activation of lecithin-cholesterol acyltransferase. J. Biol. Chem. 266:23886-23892.

29. Markwell, M.A.K., Haas, S.M., Bieber, L.L., and Tolbert, N.E. 1978. A modification of the Lowry procedure to simplify protein determination in membrane and lipoprotein samples. Anal. Biochem. 87:206-210.

30. Heeb, M.J., Kojima, Y., Greengard, J., and Griffin, J.H. 1995. Activated protein $\mathrm{C}$ resistance: molecular mechanisms based on studies using purified Gln ${ }^{506}$-factor V. Blood. 85:3405-3411.

31. Koedam, J.A., Meijers, J.C.M., Sixma, J.J., and Bouma, B.N. 1988. Inactivation of human factor VIII by activated protein C. Cofactor activity of protein S and protective effect of von Willebrand factor. J. Clin. Invest. 82:1236-1243.

32. Heeb, M.J., Mesters, R.M., Tans, G., Rosing, J., and Griffin, J.H. 1993. Binding of protein $\mathrm{S}$ to factor $\mathrm{Va}$ associated with inhibition of prothrombinase that is independent of activated protein C. J. Biol. Chem. 268:2872-2877.

33. Curtiss, L.K., and Edgington, T.S. 1982. Immunochemical heterogeneity of human plasma apolipoprotein B. I. Apolipoprotein B binding of mouse hybridoma antibodies. J. Biol. Chem. 257:15213-15221.

34. Savage, B., Saldivar, E., and Ruggeri, Z.M. 1996. Initiation of platelet adhesion by arrest onto fibrinogen or translocation on von Villebrand factor. Cell. 84:289-297.

35. Dahlbäck, B., Carlsson, M., and Svensson, P.J. 1993. Familial thrombophilia due to a previously unrecognized mechanism characterized by poor anticoagulant response to activated protein C: prediction of a cofactor to activated protein C. Proc. Natl. Acad. Sci. USA. 90:1004-1008.

36. Griffin, J.H., Evatt, B., Wideman, C., and Fernández, J.A. 1993. Anticoagulant protein $C$ pathway defective in majority of thrombophilic patients. Blood. 82:1989-1993.

37. Kalafatis, M., Bertina, R.M., Rand, M.D., and Mann, K.G. 1995. Characterization of the molecular defect in factor VR506Q. J. Biol. Chem. 270:4053-4057.

38. Griffin, J.H., Gruber, A., and Fernández, J.A. 1992. Reevaluation of total, free, and bound protein S and C4b-binding protein levels in plasma anticoagulated with citrate or hirudin. Blood. 79:3203-3211.

39. de Ronde, H. and Bertina, R.M. 1994. Laboratory diagnosis of APC-resistance: a critical evaluation of the test and the development of diagnostic criteria. Thromb. Haemost. 72:880-886.

40. Sun, X., Evatt, B., and Griffin, J.H. 1994. Blood coagulation factor Va abnormality associated with resistance to activated protein $\mathrm{C}$ in venous thrombophilia. Blood. 83:3120-3125.

41. Coller, B.S., et al. 1987. Deficiency of plasma protein S, protein C, or antithrombin III and arterial thrombosis. Arteriosclerosis. 7:456-462.

42. Wiesel, M.-L., et al. 1990. Screening of protein S deficiency using a functional assay in patients with venous and arterial thrombosis. Thromb. Res. 58:461-468.

43. Köller, H., et al. 1994. Deficiency of both protein C and protein S in a family with ischemic strokes in young adults. Neurology. 44:1238-1240.

44. Folsom, A.R., Wu, K.K., Rosamond, W.D., Sharrett, A.R., and Chambless, L.E. 1997. Prospective study of hemostatic factors and incidence of coronary heart disease: the Atherosclerosis Risk in Communities (ARIC) Study. Circulation. 96:1102-1108.

45. Folsom, A.R., et al. 1998. Prospective study of markers of hemostatic function, inflammation, and endothelial function with risk of ischemic stroke. Circulation. 98:I-207. (Abstr.)

46. Kawasaki, T., et al. 1997. Hypercholesterolemia as a risk factor for deep vein thrombosis. Thromb. Res. 88:67-73.

47. Smirnov, M.D., and Esmon, C.T. 1994. Phosphatidylethanolamine incorporation into vesicles selectively enhances Factor Va inactivation by activated protein C. J. Biol. Chem. 269:816-819.

48. Wallentin, L. and Sundin, B. 1985. HDL 2 and $\mathrm{HDL}_{3}$ lipid levels in coronary artery disease. Atherosclerosis. 59:131-136.

49. Groener, J.E., Scheek, L.M., van Ramshorst, E., Krauss, X.H., and van tol, A. 1998. Delayed increase in high density lipoproteinphospholipids after ingestion of a fat load in normolipidemic patients with coronary artery disease. Arteriosclerosis. 137: 311-319.

50. Kunz, F., Pechlaner, C., Erhart, R., Fend, F., and Mühlberger, V. 1994. HDL and plasma phospholipids in coronary artery disease. Arterioscler. Thromb. 14:1146-1150.

51. Fournier, N., et al. 1997. HDL phospholipid content and composition as a major factor determining cholesterol efflux capacity from Fu5AH cells to human serum. Arterioscler. Thromb. Vasc. Biol. 17:2685-2691.

52. Pajkrt, D., et al. 1997. Differential effects of reconstituted high-density lipoprotein on coagulation, fibrinolysis and platelet activation during human endotoxemia. Thromb. Haemost. 77:303-307.

53. Rota, S., McWilliam, N.A., Baglin, T.P., and Byrne, C.D. 1998. Atherogenic lipoproteins support assembly of the prothrombinase complex and thrombin generation: modulation by oxidation and vitamin E. Blood. 91:508-515

54. Moyer, M.P., et al. 1998. Plasma lipoproteins support prothrombinase and other procoagulant enzymatic complexes. Arterioscler. Thromb. Vasc. Biol. 18:458-465.

55. Silveira, A., et al. 1994. Activation of coagulation factor VII during alimentary lipemia. Arterioscler. Thromb. 14:60-69.

56. Miller, G.J., et al. 1996. Activation of factor VII during alimentary lipemia occurs in healthy adults and patients with congenital factor XII or factor XI deficiency, but not in patients with factor IX deficiency. Blood. 87:4187-4196.

57. Cochrane, C.G., and Griffin, J.H. 1980. The biochemistry and pathophysiology of the contact system of plasma. Adv. Immunol. 33:241-306.

58. Bradley, W.A., and Gianturco, S.H. 1988. Vitamin K-dependent proteins bind to very low-density lipoproteins. Semin. Thromb. Hemost. 14:253-257.

59. De Sousa, J.C., et al. 1988. Association between coagulation factors VII and X with triglyceride rich lipoproteins. J. Clin. Pathol. 41:940-944.

60. Xu, N., Dahlbäck, B., Öhlin, A.-K., and Nilsson, Å. 1998. Association of vitamin $\mathrm{K}$-dependent coagulation proteins and $\mathrm{C} 4 \mathrm{~b}$ binding protein with triglyceride-rich lipoproteins of human plasma. Arterioscler. Thromb. Vasc. Biol. 18:33-39. 